\title{
Effects of biophysical stimulation in patients undergoing arthroscopic reconstruction of anterior cruciate ligament: prospective, randomized and double blind study
}

\author{
Francesco Benazzo - Giacomo Zanon - Luigi Pederzini - Fulvio Modonesi • \\ Carlo Cardile · Francesco Falez · Luigi Ciolli · Filippo La Cava • \\ Sandro Giannini · Roberto Buda - Stefania Setti - Gaetano Caruso • \\ Leo Massari
}

Received: 28 November 2007 / Accepted: 28 February 2008/Published online: 2 April 2008

(C) The Author(s) 2008

\begin{abstract}
Pre-clinical studies have shown that treatment by pulsed electromagnetic fields (PEMFs) can limit the catabolic effects of pro-inflammatory cytokines on articular cartilage and favour the anabolic activity of the chondrocytes. Anterior cruciate ligament (ACL) reconstruction is usually performed by arthroscopic procedure that, even if minimally invasive, may elicit an inflammatory joint reaction detrimental to articular cartilage. In this study the effect of I-ONE PEMFs treatment in patients undergoing ACL reconstruction was investigated. The study end-points were (1) evaluation of patients'
\end{abstract}

F. Benazzo · G. Zanon

IRCCS Foundation, Orthopaedic and Traumatology Department,

S. Matteo Hospital Institute, University of Pavia, Pavia, Italy

L. Pederzini · F. Modonesi

Orthopaedic Department of Nuovo Ospedale di Sassuolo,

Modena, Italy

C. Cardile

Orthopaedic Department of University of Milano - Bicocca,

San Gerardo Hospital, Monza, Italy

F. Falez $\cdot$ L. Ciolli $\cdot$ F. L. Cava

Orthopaedic Department of S. Spirito Hospital Institute,

Roma, Italy

S. Giannini · R. Buda

Orthopaedic Department of Rizzoli Orthopaedic Institute,

Bologna, Italy

S. Setti $(\bowtie)$

IGEA, Clinical Biophysics,

Via Parmenide 10/A, 41012 Carpi (Mo), Italy

e-mail:s.setti@igeamedical.com

G. Caruso · L. Massari

Department of Biomedical Science and Advanced Therapy,

University of Ferrara, Ferrara, Italy functional recovery by International Knee Documentation Committee (IKDC) Form; (2) use of non-steroidal antiinflammatory drugs (NSAIDs), necessary to control joint pain and inflammation. The study design was prospective, randomized and double blind. Sixty-nine patients were included in the study at baseline. Follow-up visits were scheduled at 30,60 and 180 days, followed by 2 -year follow-up interview. Patients were evaluated by IKDC Form and were asked to report on the use of NSAIDs. Patients were randomized to active or placebo treatments; active device generated a magnetic field of $1.5 \mathrm{mT}$ at $75 \mathrm{~Hz}$. Patients were instructed to use the stimulator (IONE) for $4 \mathrm{~h}$ per day for 60 days. All patients underwent ACL reconstruction with use of quadruple hamstrings semitendinosus and gracilis technique. At baseline there were no differences in the IKDC scores between the two groups. At follow-up visits the SF-36 Health Survey score showed a statistically significant faster recovery in the group of patients treated with I-ONE stimulator $(P<0.05)$. NSAIDs use was less frequent among active patients than controls $(P<0.05)$. Joint swelling resolution and return to normal range of motion occurred faster in the active treated group $(P<0.05)$ too. The 2-year follow-up did not shown statistically significant difference between the two groups. Furthermore for longitudinal analysis the generalized linear mixed effects model was applied to calculate the group $\times$ time interaction coefficient; this interaction showed a significant difference $(P<0.0001)$ between the active and placebo groups for all investigated variables: SF-36 Health Survey, IKDC Subjective Knee Evaluation and VAS. Twenty-nine patients (15 in the active group; 14 in the placebo group) underwent both ACL reconstruction and meniscectomy; when they were analysed separately the differences in SF36 Health Survey scores between the two groups were 
larger then what observed in the whole study group $(P<0.05)$. The results of this study show that patient's functional recovery occurs earlier in the active group. No side effects were observed and the treatment was well tolerated. The use of I-ONE should always be considered after ACL reconstruction, particularly in professional athletes, to shorten the recovery time, to limit joint inflammatory reaction and its catabolic effects on articular cartilage and ultimately for joint preservation.

Keywords Anterior cruciate ligament .

Chondroprotection · Biophysical stimuli .

Pulsed electromagnetic fields · Joint preservation

\section{Introduction}

Articular cartilage performs mechanical functions absorbing the different loads applied to a joint in the course of daily activity [19]. Homeostasis and mechanical competence of cartilage are regulated by the activity of the chondrocytes that maintain the function and the integrity of the extracellular matrix, proteoglycans and collagen.

In consideration of the scant repairability of the cartilage, even modest damages resulting from trauma or inflammation may be the starting point for cartilage degeneration leading over time to extensive lesions that deepen into the thickness of the cartilage itself, ultimately exposing the subchondral bone tissue $[3,17]$.

Joint injury may involve synovial tissue, cartilage and subchondral bone leading to joint inflammation, swelling and pain. Surgical interventions must certainly be included among the triggers of inflammatory reaction in a joint [12]. The development of arthroscopic procedures has undoubtedly limited joint damage associated to surgery for reconstruction of ligaments; nevertheless, it does not avoid the inflammatory response. Thus, while arthroscopic procedures make surgery less invasive, the inflammatory response at the joint remains and the release of proinflammatory cytokines in the synovial fluid is associated with an increase in the aggrecanase activities that lead to a degradation of the cartilage matrix, and also inhibit proteoglycan synthesis $[11,15,18]$. To prevent cartilage damage, current pharmacological therapies aim to control the catabolic effects of the pro-inflammatory cytokines and enhance anabolic activity, proteoglycan synthesis and proliferation of chondrocytes. Drugs that combine the above effects are called chondroprotectors; in this category should be included drugs with $\mathrm{A}_{2 \mathrm{~A}}$ adenosine receptor agonist activity, able to stimulate the physiological pathways that control inflammation and promote chondrocyte anabolic activities. Nevertheless, these drugs are in early stages of clinical testing [5].
Pre-clinical studies have shown that pulsed electromagnetic fields (PEMFs) in vitro favour the proliferation of chondrocytes [6, 16], stimulate proteoglycan synthesis [7] and demonstrate an $\mathrm{A}_{2 \mathrm{~A}}$ adenosine receptor agonist activity $[20,21]$. Electromagnetic fields in vivo prevent degeneration of articular cartilage and down-regulate the synthesis and release of pro-inflammatory cytokines in the synovial fluid $[2,4,8,9]$. These findings suggest that electromagnetic fields may be used to control joint inflammation and to stimulate cartilage anabolic activities, finally resulting in chondroprotection.

A clinical study performed in patients undergoing arthroscopic treatment for cartilage lesions showed that biophysical treatment with PEMFs was well tolerated by the patients and led to a decrease in the use of non-steroidal anti-inflammatory drugs (NSAIDs) and to an early functional recovery; the positive effect of the treatment was maintained at a 3-year follow-up [22].

Arthroscopic reconstruction is the treatment of choice following anterior cruciate ligament (ACL) rupture; although minimally invasive, the procedure is associated with joint reaction involving the synovia and it is expected to lead to an increase of pro-inflammatory cytokines in the synovial fluid with catabolic effect on articular cartilage. In this study, we evaluated whether the treatment with PEMFs could be used to control joint inflammatory response in patients undergoing ACL reconstruction. The end points of the study were: (1) patients' functional recovery evaluated by International Knee Documentation Committee (IKDC) form; (2) use of NSAIDs, necessary to control joint pain and inflammation.

\section{Materials and methods}

\section{Patients and study design}

In 2004-2005, 84 patients undergoing ACL reconstruction were evaluated for inclusion in the study at five clinical centres. Of these, 69 gave their informed consent to participate in the study. The prospective randomized and double-blind study was approved by the local ethical committees. Inclusion criteria were the following: age between 18 and 45 years, ACL complete lesion following acute trauma or consequence of ligament chronic degeneration. All lesions were documented by MRI and confirmed during the intervention. The following were the exclusion criteria: osteonecrosis of the femoral condyle, rheumatoid arthritis, autoimmune disease, systemic disease and patients requiring meniscus repair.

The patients were assigned to the active or placebo group according to the following randomization criteria: age (18-30 or 31-45), sex, smoking status, origin of ACL 
rupture (traumatic or degenerative). For randomization of patients, a computer-generated schedule was prepared by a biostatistician. In this process, a random number seed was entered into the computer to generate a list that assigned equal numbers of active and placebo stimulators. The minimum number of patients per group required was calculated by power analysis taking into account the results of a previous study [22].

Of the 69 patients included, two never started the therapy, two dropped out within 2 weeks of therapy, and five did not return at follow-up visits; a total of 60 patients were therefore available for subsequent analysis. The ACL rupture occurred during sports activity in 49 patients (24 active and 25 placebo), daily activity in eight patients (four active and four placebo) and traffic accident in three patients (three active). At the time of ACL reconstruction 29 patients underwent also meniscectomy: 15 in the active group and 14 in the placebo.

\section{Clinical evaluation}

The patients were evaluated by IKDC Form before the intervention and at 30, 60 and 180 days afterwards. The different parts of the questionnaire, IKDC Current Health Assessment Form (SF-36 Health Survey), IKDC Subjective Knee Evaluation Form and IKDC Knee Examination Form were analysed separately. As regards the scores of the questionnaires, for each subject we considered the changes at follow-up visits with respect to the values recorded at baseline, before surgery.

Pain intensity was evaluated by visual analogue scale (VAS) of $10-\mathrm{cm}$ length: $0 \mathrm{~cm}$ no pain, $10 \mathrm{~cm}$ maximum pain. The patients were allowed to use NSAIDs to control knee pain when present and had to report doing so.

A 2-year follow-up telephone interview was conducted and the patients were asked: (a) if they had undergone further surgery at the knee, (b) if they had pain at the knee, (c) if they had functional limitation in daily activity, (d) if they returned to previous sport activity level.
Surgical technique

ACL arthroscopic repair was performed by quadruple hamstrings semitendinosus and gracilis technique. Tendons were harvested with the tendons stripper through a 2-3 cm vertical incision on the antero-medial tibial area. Diameter of the quadruple hamstrings semitendinosus and gracilis tendons was measured, while the tibial tunnel and same size femoral tunnel (30 mm length) were prepared. The graft was pulled up through the tibial tunnel with the knee at $90^{\circ}$ of flexion and suspended on the external femoral cortex (Endobutton, Smith and Nephew, London, UK). Distally, the graft was fixed with an interference absorbable screw at the tibia at $10^{\circ}$ of flexion.

\section{Rehabilitation}

All the patients underwent standard rehabilitation using passive knee flexion daily. Exercises started within the third post-operative day with isometric quadriceps contractions and then progressed to active closed-chain exercises by 4-6 weeks postoperatively. During the first 20 days patients were instructed to use two crutches and then progressive weight bearing until the end of the second month.

\section{Biophysical stimulation}

The patients were treated with active or placebo devices. The active stimulators (I-ONE; IGEA, Carpi, Italy) generated a magnetic field of peak intensity of $1.5 \mathrm{mT}$ at a frequency of $75 \mathrm{~Hz}$; no heat or vibration was felt by the patient during treatment (Fig. 1).

The patients were instructed to use the stimulator for $4 \mathrm{~h}$ per day, not necessarily consecutively, for 60 days. Treatment started within 7 days from the surgery. Each device contained a clock to monitor the hours of use.
Fig. 1 Left I-ONE PEMFs generator. Right wave form of magnetic field, $1.5 \mathrm{mT}$ peak value (top); electric field induced in a standard coil probe made of 50 turns $(0.5 \mathrm{~cm} \varnothing)$ of copper wire $(0.2 \mathrm{~mm} \varnothing)$, peak value $3 \mathrm{mV} / \mathrm{cm}$ (bottom)
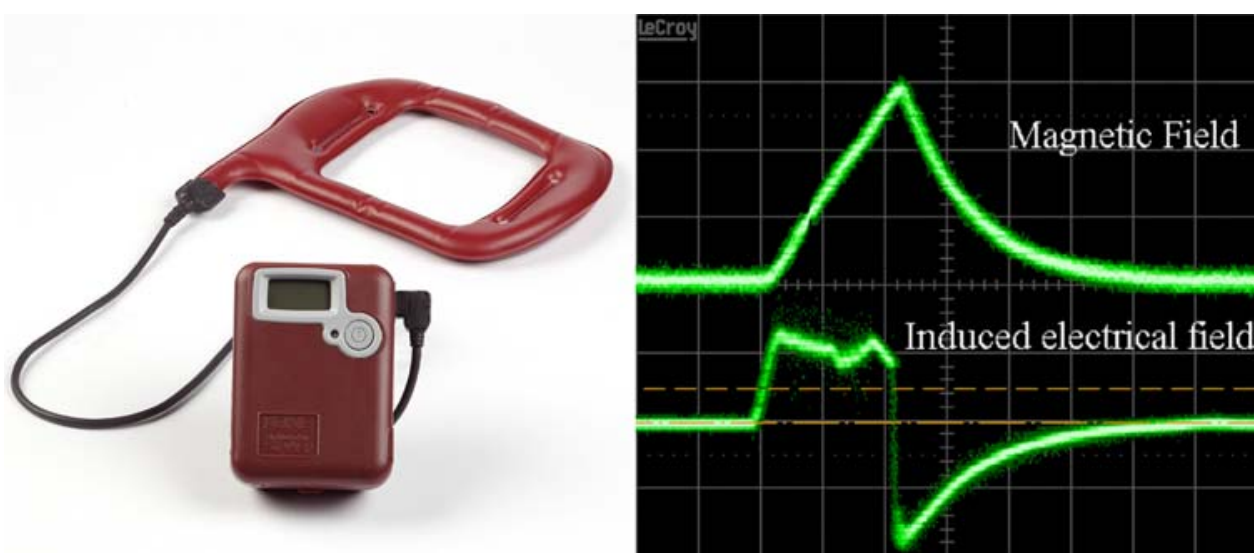
Statistical analysis

The results were analysed with SPSS 13.0 (Statistical Packages for Social Sciences Inc, Chicago, IL, USA). Comparison among the continuous variables in the two groups was performed with Student's heteroschedastic $t$ test; comparison of continuous variables within each group during follow-up was performed with Student's paired $t$ test.

Binomial and categorical variables were compared by contingency tables applying the chi-square test for $2 \times 2$ tables and the Cochran Mantel Haenszel test for larger size tables.

Generalized linear mixed effects model was applied to the SF-36 Health Survey, IKDC Subjective Knee Evaluation and VAS data to test if a different trend between the two groups was present during follow-up by correcting for the following covariates: sex, age, weight, height, hours of treatment, smoking status, use of NSAIDs. In this analysis, a mathematical model is built which takes into account the trend over time of individual patients belonging to each group (Group $\times$ Time interaction) and determines if a statistical difference exists between the groups during the follow-up [10].

The minimum significance level for all the statistical tests was set at $P<0.05$.

\section{Results}

At baseline, the two groups of study were homogeneous for age, weight, height, VAS, SF-36 Health Survey and IKDC Subjective Knee Evaluation score (Table 1).

Average daily treatment was the same in both groups: $3.92 \pm 0.5 \mathrm{~h} /$ die versus $3.13 \pm 0.3 \mathrm{~h} /$ die in the I-ONE group and the placebo group, respectively ( $P=$ n.s.).

The average pain was modest and almost absent at 6 months' follow-up: $0.7 \pm 0.2 \mathrm{~cm}$ among placebo and $0.9 \pm 0.2 \mathrm{~cm}$ among active. At 30 days, less patients in the active group used NSAIDs: $8 \%$ in the I-ONE group versus $27 \%$ in the placebo group $(P<0.05)$.

The SF-36 Health Survey score decreased significantly at 30 days, in both groups $(P<0.0005)$. At 60 days the

Table 1 Characteristics of the groups at baseline

\begin{tabular}{|c|c|c|c|c|c|}
\hline & \multicolumn{2}{|c|}{$\begin{array}{l}\text { Placebo } \\
\# 29\end{array}$} & \multicolumn{2}{|c|}{$\begin{array}{l}\text { I-ONE } \\
\# 31\end{array}$} & \multirow[t]{2}{*}{$P$} \\
\hline & Mean & SE & Mean & SE & \\
\hline Age & 29.6 & 1.6 & 32.5 & 1.4 & 0.17 \\
\hline Weight & 72 & 2 & 73 & 3 & 0.73 \\
\hline Height & 175 & 1 & 174 & 2 & 0.59 \\
\hline VAS & 2.4 & 0.3 & 3.2 & 0.5 & 0.27 \\
\hline SF-36 Health Survey & 37 & 2 & 37 & 2 & 0.95 \\
\hline $\begin{array}{l}\text { IKDC Subjective Knee Evaluation } \\
\text { Form }\end{array}$ & 48 & 3 & 47 & 3 & 0.90 \\
\hline
\end{tabular}

mean SF-36 Health Survey score in the I-ONE patients already exceeded the initial value (by 3.2 points), whereas in the patients of the placebo group SF-36 Health Survey score was slightly below the initial mean value (by -0.7 units). At 6 months a significant $(P<0.005)$ increase was observed for SF-36 Health Survey average values in both groups; the patients of the I-ONE group were above the initial values by 10.1 units, while the placebo group exceeds the baseline value by 7.2 units. The mean changes of SF-36 Health Survey score in the I-ONE group are systematically higher with respect to placebo during follow-up, $P<0.05$ (Fig. 2).

The IKDC Subjective Knee Evaluation score increased over 6 months and did not show significant differences between the two groups at any follow-up visit.

The IKDC Knee Examination Form outlined both groups including subjects with joint swelling before surgery (one in placebo and two in I-ONE group, $P=$ n.s.) and at 30 days' follow-up (five in placebo and six in I-ONE group, $P=$ n.s.). On day 60, joint swelling was observed in the placebo group (two patients) only. Joint swelling was not observed any more at 6 months' follow-up. Limitation in the passive range of motion of the knee was more frequent in the placebo group than in the I-ONE group $(P<0.05)$ (Fig. 3).

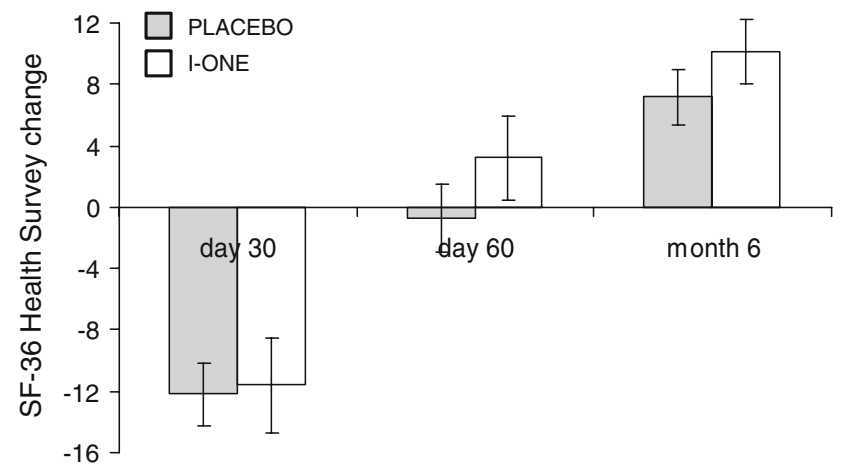

Fig. 2 Mean changes of SF-36 Health Survey $( \pm S E)$ versus baseline in the two groups $(P<0.05)$

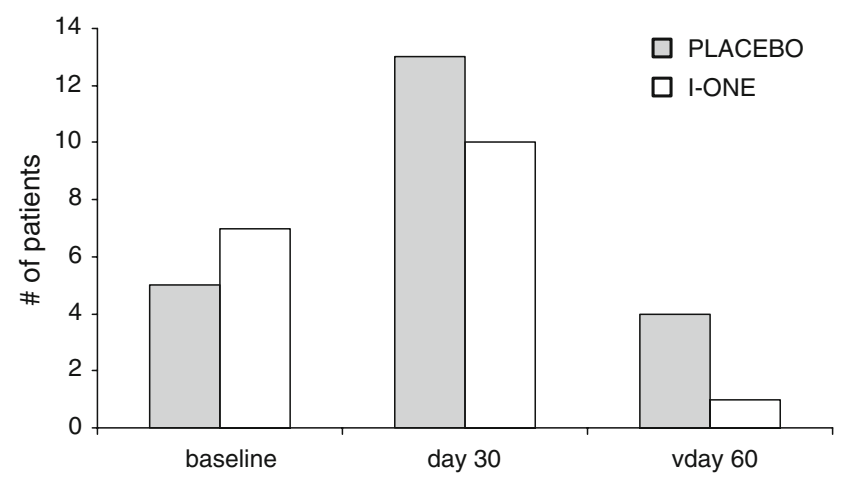

Fig. 3 Patients with limitation in passive range of motion in the two groups, $P<0.05$ 
Finally, the generalized linear mixed effects analysis revealed a significantly different trend (group $\times$ time interaction, $P<0.0001$ ) between the two groups for SF-36 Health Survey score, IKDC Subjective Knee Evaluation score and for VAS, showing a positive effect of I-ONE treatment. The estimate coefficients and significance of independent variables for three models are displayed in Table 2.

Table 2 Generalized linear mixed effects models in which the dependent variables considered are: SF-36 Health Survey score, IKDC Subjective Knee Evaluation score and VAS, respectively

\begin{tabular}{|c|c|c|c|c|}
\hline & Coefficient & Std. err. & $z$ test & $P<$ \\
\hline \multicolumn{5}{|l|}{ SF-36 Health Survey } \\
\hline Group & 0.361 & 2.690 & 0.13 & 0.893 \\
\hline Hours of treatment & -0.014 & 0.008 & -1.79 & 0.073 \\
\hline Sex & -8.348 & 4.810 & -1.74 & 0.083 \\
\hline Weight & -0.423 & 0.171 & -2.48 & 0.013 \\
\hline Height & 0.220 & 0.254 & 0.87 & 0.386 \\
\hline Age & -0.212 & 0.137 & -1.55 & 0.120 \\
\hline Smoking status & 0.768 & 2.075 & 0.37 & 0.711 \\
\hline Use of NSAIDs & -4.667 & 2.726 & -1.71 & 0.087 \\
\hline Time & 0.125 & 0.034 & 4.58 & 0.000 \\
\hline Group $\times$ Time & 0.051 & 0.014 & 3.67 & 0.0001 \\
\hline Constant & 37.940 & 40.302 & 0.94 & 0.347 \\
\hline \multicolumn{5}{|c|}{ IKDC Subjective Knee Evaluation } \\
\hline Group & -1.499 & 3.417 & -0.44 & 0.661 \\
\hline Hours of treatment & -0.007 & 0.011 & -0.62 & 0.533 \\
\hline Sex & -19.390 & 6.172 & -3.14 & 0.002 \\
\hline Weight & -0.578 & 0.216 & -2.68 & 0.007 \\
\hline Height & 0.150 & 0.322 & 0.47 & 0.642 \\
\hline Age & -0.754 & 0.185 & -4.08 & 0.000 \\
\hline Smoking status & -2.214 & 2.783 & -0.80 & 0.426 \\
\hline Use of NSAIDs & -2.941 & 3.805 & -0.77 & 0.440 \\
\hline Time & 0.145 & 0.051 & 2.89 & 0.000 \\
\hline Group $\times$ Time & 0.167 & 0.031 & 5.37 & 0.000 \\
\hline Constant & 94.561 & 51.382 & 1.84 & 0.066 \\
\hline \multicolumn{5}{|l|}{ VAS } \\
\hline Group & 0.799 & 0.455 & 1.76 & 0.079 \\
\hline Hours of treatment & -0.000 & 0.001 & -0.22 & 0.824 \\
\hline Sex & 1.583 & 0.806 & 1.96 & 0.050 \\
\hline Weight & 0.020 & 0.029 & 0.67 & 0.505 \\
\hline Height & 0.027 & 0.043 & 0.64 & 0.524 \\
\hline Age & 0.033 & 0.022 & 1.5 & 0.133 \\
\hline Smoking status & 0.246 & 0.337 & 0.73 & 0.464 \\
\hline Use of NSAIDs & -0.825 & 0.434 & -1.90 & 0.058 \\
\hline Time & -0.441 & 0.244 & 3.89 & 0.000 \\
\hline Group $\times$ Time & -0.009 & 0.002 & -4.00 & 0.000 \\
\hline Constant & -5.593 & 6.721 & -0.83 & 0.405 \\
\hline
\end{tabular}

The Group $\times$ Time interaction term describes the different trend between the groups

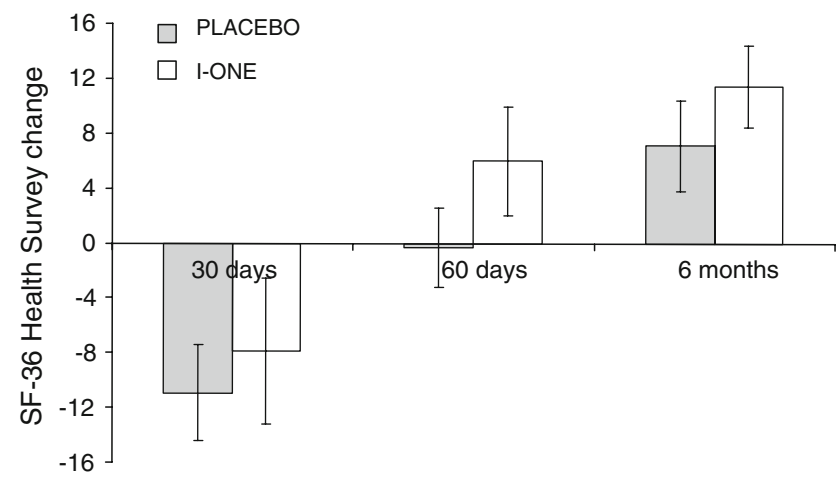

Fig. 4 Patients undergoing ACL and meniscectomy: mean changes of SF-36 Health Survey ( \pm SE) versus baseline in the two groups $(P<0.05)$

At the 2-year follow-up interview $86 \%$ of the patients in the I-ONE group and $75 \%$ in the placebo group reported complete functional recovery, no knee pain and return to sport activity.

\section{ACL reconstruction and meniscectomy}

When the cohort of patients, undergoing both ACL reconstruction and meniscectomy, was analysed separately, the SF-36 Health Survey score confirmed the faster recovery trend among I-ONE treated patients compared to placebo, $P<0.05$ (Fig. 4). At 6 months, SF-36 Health Survey average score increase was 11.4 in the I-ONE group $(P<0.005$ vs. baseline $)$ and 7.1 in placebo group $(P=\mathrm{ns}$ vs. baseline). Further, the average values of SF-36 Health Survey were significantly higher in the I-ONE group compared to the placebo $(45.2 \pm 1.5$ vs. $37 \pm 2.7$, $P<0.05$ ).

The percent of patients with limitation in the passive range of motion was lower in the I-ONE group compared to the placebo one (34\% I-ONE vs. $50 \%$ placebo at day 30 and $4 \%$ I-ONE vs. $17 \%$ placebo at day $60, P<0.05$ ).

\section{Discussion}

Arthroscopic surgery has gained a large success and led to a significant increase in its use: about 650,000 procedures are performed in the USA each year [14]. However, the access into the joint space is always associated to an inflammatory reaction that may jeopardize the benefits expected from surgery. Joint inflammation has a catabolic effect on extracellular matrix and inhibits chondrocyte activity; thus, all means capable of locally controlling the inflammation should be adopted to prevent the onset and limit the progression of cartilage damage. Furthermore, 
unlike bone tissue after damage, the cartilage will not completely recover its competence: once lost, the articular cartilage does not reform [13].

Many efforts are made to develop strategies able to control joint inflammation and to favour the anabolic activities of chondrocytes; these are challenging objectives, and up to now the pharmacological approaches based on the use of drugs, whether by systemic or by local route, have not yet been able to demonstrate a genuine chondroprotective effect in humans [19].

Pre-clinical studies have shown PEMFs to have a chondroprotective effect, mediated by the control of inflammation and by the stimulation of chondrocyte activity; thus, we hypothesized that after arthroscopic surgery PEMFs treatment can be used for articular cartilage protection and ultimately joint preservation.

This prospective, randomized and double-blind study investigated whether and to what extent the employment of I-ONE, by controlling joint reaction to arthroscopy, could accelerate functional recovery in patients undergoing ACL reconstruction. The I-ONE treatment was well tolerated by the patients and no adverse side effects were observed. The results show that, at 30 days after surgery, in I-ONE group significantly fewer patients used NSAIDs to control pain, compared to patients in the placebo group; afterwards, the use of NSAIDs was not necessary in either group.

When IKDC Subjective Knee Evaluation average scores were analysed, we found no statistically significant difference between the I-ONE and placebo group; this is in agreement with the findings of other authors who reported that this parameter does not correlate with the other clinical information collected using the SF-36 Health Survey form [1]. However, when the results of the two groups were analysed by generalized linear mixed effects model, which takes into account the trend of each patient in both groups and the effect of confounding factors, we could evidence a positive significant effect of I-ONE treatment also in the Subjective Knee Evaluation (Table 2).

The SF-36 Health Survey average scores at baseline were the same in the I-ONE and placebo groups; however, the high standard deviation testify the large distribution of initial score values. To monitor patient's recovery after ACL reconstruction, we considered the SF-36 Health Survey score changes with respect to baseline for each individual subject. At 2 and 6 months SF-36 Health Survey increase is undoubtedly higher in I-ONE group than in the placebo group. This result indicates a faster recovery in the treated patients. This positive effect of I-ONE treatment is confirmed by the generalized linear mixed effects analysis. Further, when the cohort of patients who underwent both
ACL reconstruction and meniscectomy was analysed, we observed that the average increase of SF-36 Health Survey at 60 days in the I-ONE group was the same as that of placebo group at 6 months (6.0 vs. 7.1, $P=$ n.s.).

The IKDC Knee Examination Form showed how in the placebo group the resolution of joint swelling and the recovery of complete range of motion occur later compared to the I-ONE group; no significant difference in scoring was observed among centres.

The study end-points were thus demonstrated: fewer patients in the I-ONE group required the use of NSAIDs and their functional recovery was faster.

At 2-year follow-up no statistically significant difference was observed between two groups, although the percent of patients with complete recovery was slightly higher in the I-ONE group.

In this study we applied a statistical analysis specifically developed for longitudinal studies that allows to calculate the group $\times$ time interaction. This test, that considers individual patient's score at different time points and the possible influence of confounding factors, supports the positive effect of I-ONE treatment on the recovery of patients undergoing ACL reconstruction.

Our data confirm the results reported by Zorzi et al. [22] in a group of patients treated with I-ONE following an arthroscopic treatment for cartilage lesions. To the authors' knowledge, there are no other reports of use of biophysical stimulation after surgical procedures of the knee.

Biophysical stimulation allows treating individual joints, permeating the whole cartilage surface and thickness, the synovia and the subchondral bone. The effectiveness of biophysical stimulation is not limited by considerations such as diffusion ability and concentration gradient, which are present and important in the dynamic of a pharmacological intervention; joint tissues are paramagnetic, they do not attenuate the biophysical signal and thus are all homogenously exposed to the treatment efficacy. Biophysical stimulation is an effective therapeutic intervention to control the detrimental consequences of the inflammation over articular cartilage in the absence of negative side effects.

I-ONE should always be considered after ACL reconstruction, particularly in professional athletes, to shorten the recovery time, to limit joint inflammatory reaction and ultimately for joint preservation.

Acknowledgments Supported by Igea through a grant of Research funding of Regione Emilia Romagna. Setti is Igea employ; the other authors have no conflict of interest.

Open Access This article is distributed under the terms of the Creative Commons Attribution Noncommercial License which permits any noncommercial use, distribution, and reproduction in any medium, provided the original author(s) and source are credited. 


\section{References}

1. Aglietti P, Giron F, Buzzi R, Biddau F, Sasso F (2004) Anterior cruciate ligament reconstruction: bone-patellar tendon-bone compared with double semitendinosus and gracilis tendon grafts. A prospective, randomized clinical trial. J Bone Joint Surg Am 86(10):2143-2155

2. Benazzo F, Cadossi M, Cavani F, Fini M, Giavaresi G, Setti S, Cadossi R, Giardino R (2008) Cartilage repair with osteochondral autografts in sheep: Effect of biophysical stimulation with pulsed electromagnetic fields. J Orthop Res [Epub ahead of print]

3. Buckwalter JA, Mankin HJ (1997) Articular cartilage: part I-II. J Bone Joint Surg 79(4):600-632

4. Ciombor DM, Aaron RK, Wang S, Simon B (2003) Modification of osteoarthritis by pulsed electromagnetic field-a morphological study. Osteoarthr Cartil 11(6):455-462

5. Cohen SB, Gill SS, Baer GS, Leo BM, Scheld WM, Diduch DR (2004) Reducing joint destruction due to septic arthrosis usingan adenosine2A receptor agonist. J Orthop Res 22:427-435

6. De Mattei M, Caruso A, Pezzetti F, Pellati A, Stabellini G, Sollazzo V, Traina GC (2001) Effects of pulsed electromagnetic fields on human articular chondrocyte proliferation. Connect Tissue Res 42(4):269-279

7. De Mattei M, Fini M, Setti S, Ongaro A, Gemmati D, Stabellini G, Pellati A, Caruso A (2003) Effects of electromagnetic fields on proteoglycan metabolism of bovine articular cartilage explants. Connect Tissue Res 44:154-159

8. Fini M, Giavaresi G, Torricelli P, Cavani F, Setti S, Cane V, Giardino R (2005) Pulsed electromagnetic fields reduce knee osteoarthritic lesion progression in the aged Dunkin Hartley guinea pig. J Orthop Res 23(4):899-908

9. Fini M, Torricelli P, Giavaresi G, Aldini NN, Cavani F, Setti S, Nicolini A, Carpi A (2007) Effect of pulsed electromagnetic field stimulation on knee cartilage, subchondral and epyphiseal trabecular bone of aged Dunkin Hartley guinea pigs. Biomed Pharmacother

10. Fitzmaurice GM, Laird NM, Ware JH (2004) Generalized linear mixed effects models. In: Balding DJ, Cressie NAC, Fisher NI, Johnstone IM, Kadane JB, Molenberghs G, Ryan LM, Scott DW, Smith AFM, Teugels JL, Barnett V, Hunter JS, Kendall DG (eds) Applied longitudinal analysis. Wiley, Hoboken, pp 325-358
11. Goldring SR, Goldring MB (2004) The role of cytokines in cartilage matrix degeneration in osteoarthritis. Clin Orthop Relat Res 427:S27-S36 (Review)

12. Green DM, Noble PC, Bocell JR Jr, Ahuero JS, Poteet BA, Birdsall HH (2006) Effect of early full weight-bearing after joint injury on inflammation and cartilage degradation. J Bone Joint Surg Am 88(10):2201-2209

13. Hunter W (1743) Of the structure and diseases of the articular cartilages. Philos Trans Lond 42:514-521

14. Owings MF, Kozak LJ (1998) Ambulatory and inpatient procedures in the United States, 1996. Vital Health Stat 13(139):1-119

15. Pellettier JP (1999) The influence of tissue cross-talking on OA progression: role of nonsteroidal anti-inflammatory drugs. Osteoarthr Cartil 7:374-376

16. Pezzetti F, De Mattei M, Caruso A, Cadossi R, Zucchini $P$, Carinci F, Traina GC, Sollazzo V (1999) Effects of pulsed electromagnetic fields on human chondrocytes: an in vitro study. Calcif Tissue Int 65:396-401

17. Radin EL, Rose RM (1986) Role of subchondral bone in the initiation and progression of cartilage damage. Clin Orthop 213:34-40

18. Schuerwegh AJ, Dombrecht EJ, Stevens WJ, Van Offel JF, Bridts CH, De Clerck LS (2003) Influence of pro-inflammatory (IL-1 alpha, IL-6, TNF-alpha, IFN-gamma) and anti-inflammatory (IL4) cytokines on chondrocyte function. Osteoarthr Cartil 11(9):681-687

19. Ulrich-Vinther M, Maloney MD, Schwarz EM, Rosier R, O'Keefe RJ (2003) Articular cartilage biology. J Am Acad Orthop Surg 11(6):421-430 (Review)

20. Varani K, Gessi S, Merighi S, Iannotta V, Cattabriga E, Spisani S, Cadossi R, Borea PA (2002) Effect of low frequency electromagnetic fields on A2A adenosine receptors in human neutrophils. Br J Pharmacol 136:57-66

21. Varani K, De Mattei M, Vincenzi F, Gessi S, Merighi S, Pellati A, Ongaro A, Caruso A, Cadossi R, Borea PA (2008) Characterization of adenosine receptors in bovine chondrocytes and fibroblast-like synoviocytes exposed to low frequency low energy pulsed electromagnetic fields. Osteoarthr Cartil 16(3):292-304

22. Zorzi C, Dall'oca C, Cadossi R, Setti S (2007) Effects of pulsed electromagnetic fields on patients' recovery after arthroscopic surgery: prospective, randomized and double-blind study. Knee Surg Sports Traumatol Arthrosc 15(7):830-834 